\title{
A sensitive HPLC-FLD method for the quantification of 6-O- demethylmenisporphine isolated from Menispermi Rhizoma in rat plasma
}

\author{
Jinxia Wei ${ }^{1}$, Jia Shao ${ }^{2}$, Yanan $\mathrm{Li}^{1}$ and Yubo $\mathrm{Li}^{1^{*}}$ (B)
}

\begin{abstract}
Background: To investigate the pharmacokinetics of 6-O-demethylmenisporphine, an oxoisoaporphine alkaloid with significant anti-tumor activities and isolated from Menispermi Rhizoma, a novel and sensitive HPLC assay was established for 6-O-demethylmenisporphine quantification in rat plasma.

Methods: Peak responses were detected by a highly selective and sensitive fluorescence detector with 426-nm excitation and 514-nm emission wavelengths. Curcumin was employed as the internal standard (IS). A Capcell Pak $\mathrm{C}_{18}$ column $(150 \mathrm{~mm} \times 4.6 \mathrm{~mm}$ i.d., $5 \mu \mathrm{m})$ and an isocratic elution procedure with a flow rate of $1.0 \mathrm{~mL} / \mathrm{min}$ were used to exclude the endogenous interfering substance. Acetonitrile-water $(68: 32, \mathrm{v} / \mathrm{v})$ containing $1 \%$ formic acid was employed as mobile phase. A 7-point calibration curve that covered the concentration range of 10-2500 ng/mL was constructed.

Results: A good linearity was observed with a correlation coefficient $(r)$ of 0.9993 . The lower limit of quantification for 6-O-demethylmenisporphine was $10 \mathrm{ng} / \mathrm{mL}$. The mean recoveries of analyte in rat plasma exceeded $80.5 \%$. The precision at four concentration levels was within $11.3 \%$ and the accuracy ranged from -7.6 to $6.7 \%$.

Conclusion: Using this new HPLC-FLD method, the investigation of plasma samples from rats following oral dosing of neat compound and Menispermi Rhizoma extract was successfully conducted. The results will provide a reference for the evaluation of preclinical safety of 6-O-demethylmenisporphine.
\end{abstract}

Keywords: Menispermi Rhizoma, 6-O-Demethylmenisporphine, Pharmacokinetics, HPLC-fluorescence method

\section{Introduction}

Traditional Chinese medicine has attracted widespread attention and become increasingly popular around the world for its special effects on many diseases. Menispermi Rhizoma, originating from plant rootstock of Menispermum dauricum DC. (Menispermaceae), is one of the famous traditional Chinese medicines with significant therapeutic efficacy on sore throat, enteritis, and rheumatic diseases (China Pharmacopoeia Committee,

\footnotetext{
* Correspondence: syykdxwjx410@163.com

${ }^{1}$ School of Chinese Materia Medica, Tianjin University of Traditional Chinese Medicine, 312 Anshan West Road, Tianjin 300193, China

Full list of author information is available at the end of the article
}

2015). Based on the literature reports, many biological investigations of Menispermi Rhizoma (MR) have been carried out (Wang et al., 2001; Wang et al., 2014; Yu et al., 2019). The EtOH extract has been shown to exhibit several biological activities, such as antiinflammatory, anti-tumor, and anti-arrhythmic effects; neuroprotection; inhibiting platelet aggregation; and $\mathrm{T}$ cell-related leukemia and lymphoma growth (Su et al., 2016; Wu et al., 2018; Yu et al., 2019; Zhao et al., 2012). The pharmacological activities can be attributed to a large number of active components in MR. The previous phytochemical reports on MR indicated that alkaloids were major active constituents (Peng, 2018), and a 
variety of alkaloids have been isolated and identified from MR (Li et al., 2013; Yu et al., 2019). Especially for oxoisoaporphine alkaloids, some investigations have found that the natural existence of this type of alkaloids is obviously limited to Menispermaceae species ( $\mathrm{Yu}$ et al., 2019). Oxoisoaporphine alkaloid attracts much attention from researchers due to its unique structure. Many researches demonstrated oxoisoaporphine derivatives exhibit significant activities, for example, inhibitory activity and selectivity for acetylcholinesterase (AChE), as well as higher DNA-binding affinity and powerful anti-tumor cell activities (Yu et al., 2001; Cheng et al., 2012; Zhang et al., 2018).

6-O-Demethylmenisporphine (DMP; Fig. 1), a natural oxoisoaporphine alkaloid isolated from MR, is one of the main bioactive alkaloids of MR. It is yellow solid powder with intense absorption bands in the ultraviolet and visible regions. In addition, DMP has a natural fluorescence due to the presence of a highly conjugated system in the 7H-dibenzo[de, h] quinolin-7-one moiety, and it exhibits high fluorescence intensity at $426 \mathrm{~nm}$ as excitation wavelength and $514 \mathrm{~nm}$ as emission wavelength. Previous pharmacological studies have demonstrated that DMP exhibits clear anti-tumor effects on a series of cancer cells, including MCF-7, H-460, HT-29, and CEM cells (Cheng et al., 2012). Our previous study demonstrated that DMP exhibits a remarkably protective effect on hypoxia injury of EAhy 926 cells (Shao et al., 2019). Based on the significant biological activity of DMP, it could be a potential drug candidate. Despite the fact that many candidate drugs exhibit obvious pharmacological activities in vitro, they might fail due to their poor pharmacokinetic properties in vivo. Thus, pharmacokinetics study of active constituents is of great significance to the development of new drugs, which might be seen as a bridge connecting the clinical efficacy and the chemical components, and furthermore stimulates studies of the elucidation of the mechanism of drug effect and the guidance of clinical application (Lou et al., 2019). To our knowledge, many analytical techniques have been applied to the pharmacokinetics study of bioactive components, including liquid chromatography (LC) coupled with evaporative light scatting (ELSD), diode array (DAD), ultraviolet (UV), fluorescence (FLD), and mass spectrometry (MS) detectors, etc. Among them, ELSD, DAD, and UV spectroscopic methods are not sensitive enough to quantify trace amounts of DMP in biological matrix. Both FLD and MS detectors have been proved to possess high sensitivity and specificity. Fluorescence can be detected with very high sensitivity from single molecules. Meanwhile, the FLD technique is simple and rapid and requires relatively cheap instruments and relatively low maintenance costs compared with MS, and it provides a variety of merits over other analytical techniques. At present, it has been used in many chemical and biochemical applications.

There have been no reports on the determination of DMP using a HPLC-FLD method up till now, and the comparative pharmacokinetic characteristics of DMP have not yet been clarified. Thus, in our research, a novel and sensitive high-performance liquid chromatography-fluorescence detection (HPLC-FLD) method for the quantification of DMP in biological matrices has been established for the first time. Considering the importance of pharmacokinetic data for safety and risk evaluation of new drugs and the complex nature of various ingredients contained in TCM, the pharmacokinetic data on DMP following administration of neat compound and MR extract are presented and compared by employing the newly established method. The present study could be helpful for the further druggability and dosage form studies of DMP, and it provided a reliable theoretical basis and clinical guidance for rational and effective use of DMP and MR.

\section{Experimental}

\section{Materials and chemicals}

Menispermi Rhizoma was obtained from Anguo Chinese medicine wholesale market, Hebei Province, China (place of origin: Liaoning Province), and authenticated by Prof. Tianxiang Li (Department of Pharmacognosy,<smiles>COc1ccc(/C=C/C(O)=C/C(=O)/C=C/c2ccc(O)c(OC(C)(C)C)c2)cc1OC</smiles>

Fig. 1 Chemical structures of 6-O-demethylmenisporphine and curcumin (IS) 
Tianjin University of Traditional Chinese Medicine). A voucher specimen (No. 20190701) has been deposited in the Department of Pharmaceutical Analysis, Tianjin University of Traditional Chinese Medicine. The standard of DMP was isolated in the author's laboratory. The procedure for isolation, purification, and characterization of DMP as well as the corresponding spectral and physicochemical data (MS, ${ }^{1} \mathrm{H}$-NMR, and ${ }^{13} \mathrm{C}$-NMR) have been published by the author (Shao et al., 2019; Wei et al., 2016). Based on these data as well as the literature report (Li et al., 2013), the structure of DMP was accurately characterized. HPLC-DAD and HPLC-MS analysis showed that the purity was $98.10 \%$. Curcumin (HPLC purity $99.00 \%$ ) was provided by China Food and Drug Inspection and Research Institute (Beijing, China). Analytical grade acetonitrile and methanol were provided by Tianjin Concord Technology Co., Ltd., China. Formic acid, acetic acid, hydrochloric acid, and tetrahydrofuran (HPLC-grade) were obtained from Kermel Chemical Reagent Co., Ltd. (Tianjin, China). Dimethyl sulfoxide (DMSO) and polyethylene glycol 400 (PEG-400) of analytical grade were obtained from Sigma-Aldrich (St. Louis, MO, USA). Water (HPLC-grade) was purified by SZ97 ultrapure water preparation device (Shanghai Yarong Biochemical Instrument Factory, China).

\section{HPLC-FLD conditions}

The quantification of DMP in biological samples was conducted on a JASCO HPLC system (JASCO Corporation, Japan) involving two JASCO-PU-2080 plus pumps, an auto-sampler, and an Agilent 1100 fluorescence detector (Agilent Technologies, USA). A Capcell Pak $\mathrm{C}_{18}$ column $(150 \mathrm{~mm} \times 4.6 \mathrm{~mm}$ i.d., $5 \mu \mathrm{m})$ was used with an isocratic mobile phase (delivered at $1.0 \mathrm{~mL} / \mathrm{min}$ ) consisting of acetonitrile and water $(68: 32, \mathrm{v} / \mathrm{v}$, containing $1 \%$ formic acid). The column temperature was set at $25^{\circ} \mathrm{C}$. Injection volume was $20 \mu \mathrm{L}$, and the maximum response for DMP was obtained through a fluorescence detector with 426-nm excitation and 514-nm emission wavelengths. The excitation and emission wavelengths for curcumin (IS) are the same as those of DMP.

\section{Preparation of MR extract and determination of DMP in MR extract}

Ten volumes of extracting solvent were added to $100 \mathrm{~g}$ MR powder. The mixture was extracted by refluxing twice with ethanol-water $(95: 5, \mathrm{v} / \mathrm{v})$ and twice with ethanol-water $(75: 25, \mathrm{v} / \mathrm{v})$ ( $1 \mathrm{~h}$ per time). And the extracts were filtered, combined, and further evaporated to dryness under reduced pressure. An external standard method with the chromatographic condition as described above was used for the quantification of DMP in MR extract. Two hundred milligrams of MR extract was accurately weighted and dissolved in methanol in a
10-mL volumetric flask. After filtration using a 0.45$\mu \mathrm{m}$ membrane filter, the solution was injected into HPLC-FLD for analysis.

\section{Preparation of solutions}

The primary solution of DMP was prepared in $5 \mathrm{~mL}$ of tetrahydrofuran and diluted with methanol to the desired concentration of $0.1 \mathrm{mg} / \mathrm{mL}$. The DMP working solutions with concentrations that ranged from 10 to $2500 \mathrm{ng} / \mathrm{mL}$ were harvested by diluting a solution of DMP with methanol. Curcumin (IS) stock solution with the concentration of $0.1 \mathrm{mg} / \mathrm{mL}$ was obtained in a similar manner, then it was diluted with methanol to get $50 \mu \mathrm{g} / \mathrm{mL}$ IS working solution.

Standard curves of DMP were obtained by evaporating $100 \mu \mathrm{L}$ of working solution to dryness with a stream of $\mathrm{N}_{2}$ at $35^{\circ} \mathrm{C}$ and mixing with $100 \mu \mathrm{L}$ blank biomatrices. The final concentrations were 10, 25, 100, 250, 500, 1500 , and $2500 \mathrm{ng} / \mathrm{mL}$. QC samples were independently obtained at a final concentration of 10 (LLOQ), 30 (lowQC), 200 (mid-QC), and $2000 \mathrm{ng} / \mathrm{mL}$ (high-QC).

Owing to the relatively high lipid solubility, DMP and MR extract were extremely difficult to dissolve in water or $0.5 \%$ carboxymethyl cellulose sodium solution (CMC$\mathrm{Na}$ ). By searching relevant literatures, a method for the preparation of dosage form of DMP and MR extract was confirmed (Shen et al., 2019; Wang et al., 2013; Yoon et al., 2020). DMP and MR extract were firstly dissolved in DMSO, then diluted with a mixture of PEG-400 and water, respectively. Finally, the volume ratio of the three solvents is 5:45:50 (DMSO:PEG-400:water).

\section{Processing of samples}

Twenty microliters of IS solution $(50 \mu \mathrm{g} / \mathrm{mL})$ was transferred into a $10-\mathrm{mL}$ clean glass tube and dried with a stream of $\mathrm{N}_{2}$ at $35^{\circ} \mathrm{C}$, and then $100 \mu \mathrm{L}$ plasma and $20 \mu \mathrm{L} 0.5 \mathrm{~mol} / \mathrm{L}$ hydrochloric acid solution were added. After vortexing for $1 \mathrm{~min}, 3 \mathrm{~mL}$ ethyl acetate was added to the extracted analyte from plasma. The mixture was shaken again for $3 \mathrm{~min}$ and centrifugated at $12,000 \times \mathrm{g}$ for $10 \mathrm{~min}$, then the supernatant was collected and dried in a water bath $\left(35^{\circ} \mathrm{C}\right)$ under $\mathrm{N}_{2}$. The dried residue was reconstituted with $200 \mu \mathrm{L} 50 \%$ acetonitrile, then vortexed for $1 \mathrm{~min}$, and centrifugated for $10 \mathrm{~min}$ at 12 , $000 \times g$, and $20 \mu \mathrm{L}$ supernatant was analyzed.

\section{Method validation}

According to the guidelines for Industry on Bioanalytical Method Validation (U.S. Food and Drug Administration , 2018), validation of the method was conducted.

\section{Specificity}

In order to evaluate the specificity of the method, blank plasma from six different batches, QC samples at LLOQ, 
and the actual sample obtained following intragastric dosing of MR extract were analyzed to exclude the interference peaks. Carryover was estimated by injecting blank plasma in six replicates following injection of an ULOQ sample.

\section{Linearity and sensitivity}

In order to evaluate linearity, a 7-point calibration curve was run in duplicate during each of the 3 days of the validation. Using weighted least-squares linear regression $\left(1 / x^{2}\right.$ as the weighting factor), the calibration curve was described by analyzing raw data (plot DMP-to-IS ratio versus DMP concentrations). Limit of detection (LOD) was defined as signal-to-noise above 3 . LLOQ was defined as signal-to-noise above 10, which is the lowest concentration of calibration curve with an acceptable precision $<20 \%$ and accuracy within $\pm 20 \%$.

\section{Accuracy and precision}

Simulated biological samples were analyzed on the same day and on three consecutive days to assess intra-assay and inter-assay accuracy and precision, which were conducted following the standard calibration curve. Each analytical run consisted of LLOQ and three QC samples in six replicates, and the concentrations for DMP were determined using the calibration curve. The percentage relative standard deviation (RSD\%) was measured to evaluate the precision, which was expected to be less than $15 \%$. Accuracy was expressed by relative error (RE\%), the criteria of which was within $\pm 15 \%$. For LLOQ, the RSD\% and $\mathrm{RE} \%$ should not exceed $\pm 20 \%$.

\section{Extraction recovery and matrix effect}

The recovery of DMP was estimated by comparing the responses of DMP in the extracted QC samples with that of samples spiked post-extraction at corresponding concentration. Matrix effect was estimated by the ratio of the responses of DMP in post-extracted blank matrices to that of pure DMP standard solutions at corresponding concentration. The above two values of IS were estimated in the same way. All analyses of recovery and matrix effect were carried out at three QC levels in six replicates.

\section{Stability}

Three aliquots of QC samples were used to estimate the experimental stability of DMP under various conditions. Different storage conditions included three freeze-thawing cycles, storage at ambient temperature for $8 \mathrm{~h}$ (QC samples), QC samples kept at $-20^{\circ} \mathrm{C}$ for at least 2 weeks, and post-preparative samples stored in the auto-sampler at $4{ }^{\circ} \mathrm{C}$ for $8 \mathrm{~h}$. RSD within $15 \%$ was considered reliable. The stability of standard solutions was evaluated by comparing the concentration of freshly prepared with that of solution stored at $4{ }^{\circ} \mathrm{C}$ for 2 weeks.

\section{Dilution effects}

When the concentrations of actual samples exceed ULOQ, it is necessary to dilute with blank plasma. In order to validate the dilution process, five- and twentytimes dilution of plasma samples were assessed in six duplicates. The simulated plasma biosamples with concentrations of 1000 and $4000 \mathrm{ng} / \mathrm{mL}$ of DMP were diluted with blank plasma matrices to get samples with concentrations of $200 \mathrm{ng} / \mathrm{mL}$.

\section{Pharmacokinetics study}

The validated HPLC-FLD method was examined by exploring the pharmacokinetic characteristics of DMP in rats. Twelve SPF-grade male Sprague-Dawley rats weighing 180-220 g were housed for 1 week under an air-conditioned environment with a temperature at $23-27^{\circ} \mathrm{C}$ and relative humidity of $40-60 \%$. This research was approved by the Tianjin University of Traditional Chinese Medicine Animal Ethics Committee (Tianjin, China) and carried out according to the ethical guidelines. Animals were fasted for at least 12 $h$ prior to oral administration of DMP and MR extract, with free access to water. Twelve animals were randomly separated into two groups for oral administration of DMP and MR extract, respectively. After oral dosing of DMP $(1 \mathrm{mg} / \mathrm{kg})$ and MR extract $(1.605$ $\mathrm{g} / \mathrm{kg}$, a dose equivalent to $1 \mathrm{mg} / \mathrm{kg} \mathrm{DMP}), 0.25 \mathrm{~mL}$ of blood samples from the suborbital vein was collected into heparinized $0.5-\mathrm{mL}$ tubes at $0,5,10,20$, and 45 min and then at $1,2,3,4,5,8,12,24,36$, and $48 \mathrm{~h}$. The plasma sample $(100 \mu \mathrm{L})$ was achieved after centrifugation at $12,000 \times g$ for $8 \mathrm{~min}$ and frozen at $20^{\circ} \mathrm{C}$ before analysis.

Drug and statistics (DAS) 3.2 and SPSS 16.0 software were employed to calculate and compare the pharmacokinetic parameters harvested following oral dosing of DMP neat substance and MR extract. A noncompartmental model was employed to calculate parameters as described previously (Balla et al., 2018). An independent sample $t$ test was used to compare the $C_{\max }$ (peak plasma concentration), $\mathrm{AUC}_{0-\infty}, \mathrm{AUC}_{0-t}$ (area under the plasma concentration-time curve). And, the $t_{1 / 2}$ (terminal elimination half-life), $\mathrm{MRT}_{0-t}, \mathrm{MRT}_{0-\infty}$ (mean residence time), Clz/F (apparent oral clearance), and $T_{\max }$ (time to reach peak plasma concentration) were analyzed via a Mann-Whitney nonparametric statistical test (Huo et al., 2013; Zhao et al., 2011). It was considered to be statistically significant when the $p$ value was below 0.05 . 


\section{Results and discussion} HPLC-FLD assay development

With the Capcell Pak $\mathrm{C}_{18}$ column operating at ambient temperature, both acetonitrile/water and methanol/water mobile phases were tried to provide effective separation of the analyte. The results showed that they all produced good resolution due to the high selectivity of the fluorescence detector. In consideration of the higher column performance, acetonitrile-water was finally used. In addition, due to the occurrence of a phenolic hydroxyl functional group in the structure of DMP, a peak with poor symmetry and serious tailing was produced no matter what types and proportions of mobile phase were tested. Therefore, it was necessary to add additives to reduce peak tailing by the suppression of the analyte ionization. Both acetic acid $(0.1 \%, 0.5 \%$, and $1 \%)$ and
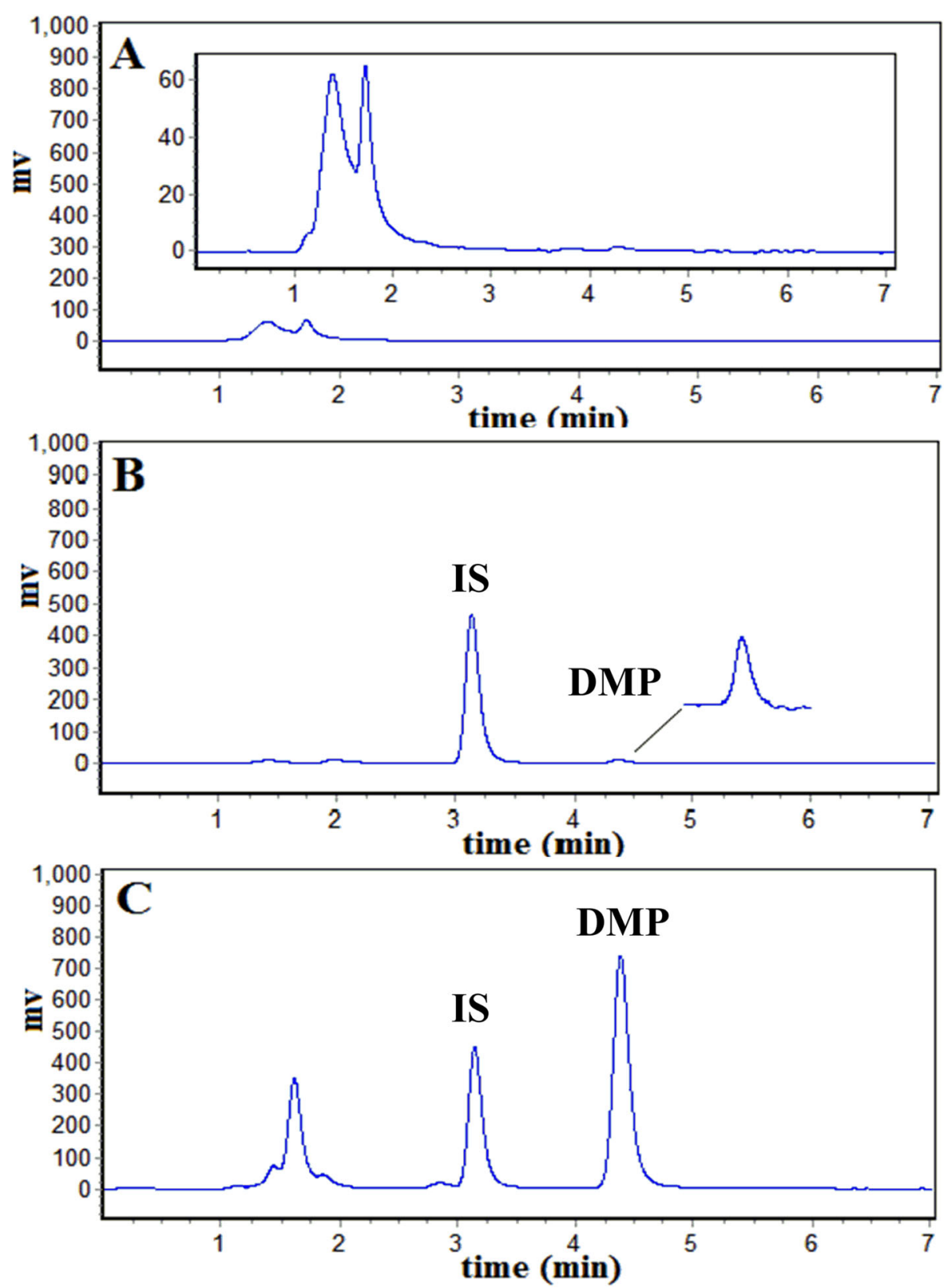

Fig. 2 The representative chromatograms for DMP and curcumin (IS) in rat plasma: a blank plasma; b blank plasma sample spiked with DMP (10 $\mathrm{ng} / \mathrm{mL}$ ) and curcumin as internal standard (IS); c plasma sample obtained at $20 \mathrm{~min}$ after oral administration of $1.605 \mathrm{~g} / \mathrm{kg}$ MR extract (DMP $1963.54 \pm 902.27 \mathrm{ng} / \mathrm{mL}$; IS $10 \mu \mathrm{g} / \mathrm{mL}$ ) 
formic acid $(0.1 \%, 0.5 \%$, and $1 \%)$ were tried as peak modifier of mobile phase. A good peak shape with acceptable tailing was obtained when using formic acid as the tailingsuppressing reagent and this may be attributed to its stronger acidity. Finally, the optimal mobile phase was acetonitrile-water $(68: 32, \mathrm{v} / \mathrm{v})$ containing $1 \%$ formic acid.

DMP exhibits ultraviolet absorption and native fluorescence due to the presence of conjugation in its structure. In the case of UV detection, $253 \mathrm{~nm}$ and $422 \mathrm{~nm}$ were tested as the maximum absorption wavelengths of DMP. It was found that interference peaks from some endogenous substances and absorbed components were observed when UV detection was used. In addition, the poor sensitivity could result in a failure to quantify trace amounts of DMP in biomatrices. The use of the more sensitive and selective spectrofluorimetric detection based on the measurement of the native fluorescence of DMP was considered for the analysis. The optimal excitation and emission wavelengths of DMP were at 426 $\mathrm{nm}$ and $514 \mathrm{~nm}$, respectively, which provided a good separation with no significant interference.

\section{Selection of internal standard (IS)}

According to FDA guidelines, IS should be a compound with a similar structure to the analyte or isotopically labeled compound. In the case of not very common compounds (such as DMP), it is very hard to find and buy its analogs. In the present paper, several compounds, such as curcumin, carbamazepine, and emodin, were investigated as an internal standard. Curcumin was finally chosen as the most appropriate IS due to its high response, excellent peak shape, stable extraction recovery, and suitable resolution of the analyte and endogenous substances.

\section{Optimization of extraction method}

A sample pre-treatment method not only can remove interferences but also can produce high extraction recovery with simple processing steps. Considering the high cost of solid phase extraction (SPE), liquid-liquid extraction (LLE) and protein precipitation (PPT) were investigated in our study. As a result, LLE could provide a much cleaner sample. Different kinds of extraction reagents, such as methyl tert-butyl ether, dichloromethane, or ethyl acetate, were tested. Among them, ethyl acetate was proved to be the best extractant giving the highest extraction efficiency. Considering the presence of a phenolic hydroxyl group in DMP, plasma samples were acidified prior to extraction to improve the extraction yield. The concentrations of hydrochloric acid solution from 0.5 to $1.5 \mathrm{~mol} / \mathrm{L}$ were adopted to identify extraction yield improvements. Finally, adding $20 \mu \mathrm{L} 0.5 \mathrm{~mol} / \mathrm{L}$ hydrochloric acid solution into $100 \mu \mathrm{L}$ plasma and processing with $3 \mathrm{~mL}$ ethyl acetate can achieve the optimum recovery over $80 \%$ for analyte.

\section{Method validation Specificity}

Figure 2 displays the respective chromatograms of blank plasma, a quality control (QC) sample with DMP at a lower limit of quantification (LLOQ) and IS, and an actual plasma at $20 \mathrm{~min}$ after dosing of MR extract. No significant endogenous peaks from matrices were found in the retention positions of DMP (4.3 min) and IS (3.2 min), indicating that the sample preparation procedure showed good efficiency and the analytical method had good specificity. The responses of DMP and IS observed in blank samples were below $5 \%$ of that observed in the LLOQ sample, demonstrating that the carryover can be neglected.

\section{Linearity and LLOQ}

A typical calibration curve equation was $y=(0.00249 \pm$ $0.000121) x+(0.00408 \pm 0.000198)(n=3$; mean \pm standard deviation) with the correlation coefficient $(r)>$ 0.9993, indicating that the curve for DMP was linear ranging from 10 to $2500 \mathrm{ng} / \mathrm{mL}$. The LOD for DMP, defined as $\mathrm{S} / \mathrm{N} \geq 3$, was calculated to be $3.0 \mathrm{ng} / \mathrm{mL}$. The LLOQ for DMP, defined as $\mathrm{S} / \mathrm{N} \geq 10$, was calculated to be $10 \mathrm{ng} / \mathrm{mL}$ with a precision of $7.6 \%$ and accuracy within - 7.1 6.7\%, respectively, which well met the pharmacokinetic requirements of DMP in biological matrices.

\section{Accuracy and precision}

Table 1 shows the accuracy and precision data of DMP obtained by detecting QC samples at four levels $(10,30$, 200 , and $2000 \mathrm{ng} / \mathrm{mL}$ ). The intra- and inter-day precisions (RSD\%) for QC samples were within 5.4\% and $11.3 \%$, respectively, and the accuracy (RE\%) for all the

Table 1 Summary of precision, accuracy, recovery, and matrix effect for DMP in rat plasma $(n=6)$

\begin{tabular}{|c|c|c|c|c|c|c|}
\hline \multirow[t]{2}{*}{ Concentration (ng/mL) } & \multicolumn{2}{|c|}{ Precision (RSD\%) } & \multicolumn{2}{|c|}{ Accuracy (RE\%) } & \multirow[t]{2}{*}{ Matrix effect (\%) } & \multirow[t]{2}{*}{ Recovery (\%) } \\
\hline & Intra-day & Inter-day & Intra-day & Inter-day & & \\
\hline 10.0 & 5.9 & 7.6 & 6.7 & -7.1 & - & - \\
\hline 30.0 & 7.7 & 10.9 & -7.6 & -1.4 & $109.1 \pm 7.8$ & $83.0 \pm 2.0$ \\
\hline 200.0 & 7.4 & 8.7 & 1.2 & 5.1 & $110.7 \pm 3.7$ & $80.5 \pm 3.0$ \\
\hline 2000.0 & 5.4 & 11.3 & -7.0 & 0.9 & $110.2 \pm 2.4$ & $82.4 \pm 3.1$ \\
\hline
\end{tabular}


Table 2 Stability results of DMP in rat plasma under different storage conditions $(n=3)$

\begin{tabular}{|c|c|c|c|c|c|c|c|c|}
\hline \multirow{2}{*}{$\begin{array}{l}\text { Concentration } \\
\text { of DMP }(\mathrm{ng} / \mathrm{mL})\end{array}$} & \multicolumn{2}{|c|}{ Room temperature for $8 \mathrm{~h}$} & \multicolumn{2}{|c|}{ Storage at $-20^{\circ} \mathrm{C}$ for 2 weeks } & \multicolumn{2}{|c|}{ Three freeze-thaw cycles } & \multicolumn{2}{|c|}{ Post-preparation stability } \\
\hline & RE\% & RSD\% & RE\% & RSD\% & RE\% & RSD\% & RE\% & RSD\% \\
\hline 30.0 & -7.9 & 6.4 & -1.9 & 7.4 & 6.6 & 4.7 & -5.7 & 7.0 \\
\hline 200.0 & -0.7 & 4.6 & -4.3 & 2.6 & -10.2 & 6.5 & -4.3 & 5.6 \\
\hline 2000.0 & -0.5 & 7.6 & -8.1 & 2.6 & -7.5 & 3.6 & -6.9 & 2.2 \\
\hline
\end{tabular}

QC levels of analyte ranged from - 7.6 to 5.1\%. The results obtained indicated that the precision and accuracy were within the acceptable criteria $( \pm 15 \%)$ of biological analysis and met the U.S. FDA guidance.

\section{Recovery and matrix effect}

DMP recovery in rat plasma at three examined QC concentrations ranged from 80.5 to $83.0 \%$ (Table 1). Matrix effects of DMP were determined to be in the range of 109.1 to $110.7 \%$. The mean recovery ratio and matrix effect of curcumin were $93.3 \pm 9.5 \%$ and $92.6 \pm 5.4 \%$ at a concentration of $10 \mu \mathrm{g} / \mathrm{mL}$, respectively. The results demonstrated that the extraction method could efficiently extract analyte from biomatrices and no significant matrices suppression or enhancement occurred during HPLC-FLD analysis.

\section{Stability}

The stability results of DMP including long-term stability (samples kept at $-20^{\circ} \mathrm{C}$ for at least 2 weeks), short-term stability (samples stored at ambient temperature for $8 \mathrm{~h}$ ), freeze-thaw stability (three freeze-thawing cycles), and post-preparative stability ( $8 \mathrm{~h}$ in the auto-sampler at $4{ }^{\circ} \mathrm{C}$ ) are displayed in Table 2. The relative error (RE\%) of DMP was well within the acceptance limit $( \pm 15 \%)$ for all the QC samples $(-8.1$ to $-1.9 \%$ for long-term stability, -7.9 to $-0.5 \%$ for short-term stability, -10.2 to $6.6 \%$ for three freeze-thaw cycles, and -6.9 to $-4.3 \%$ for post-preparation stability), which indicated that no significant degradation of the analyte was observed during the whole analysis process. The standard solutions of DMP and IS stored at $4{ }^{\circ} \mathrm{C}$ were proved to be stable for at least 2 weeks with the accuracy within $\pm 5 \%$.

\section{Dilution effects}

The dilution reliability of plasma samples diluted five and twenty times with blank plasma has been validated. The results showed that accuracy and precision determined at each concentration were within the acceptable criteria ( $\mathrm{RSD}<15 \%$; RE $\pm 15 \%$ ). The samples with concentrations above the upper limit of quantitation (ULOQ) can be fulfilled by dilution with blank plasma.

\section{Pharmacokinetics study}

Comparative pharmacokinetics after oral (p.o.) administration of DMP and MR extract were carried out using the newly established HPLC-FLD method. A summary of the corresponding pharmacokinetic parameters is given in Table 3. Plasma drug concentration-time curves for DMP have been shown in Fig. 3. The results indicated that plasma concentration of DMP was increased slowly and the maximum concentration following oral dosing of neat compound and MR extract reached 2232.3 and $3407.5 \mathrm{ng} / \mathrm{mL}$, respectively. The $T_{\max }$ for MR extract $(0.85$ h, Fig. $3 \mathrm{c})$ was observed to be quicker than that for a single substance $(2.8 \mathrm{~h}$, Fig. 3)b. Then, the DMP underwent slow elimination with an elimination half-life $\left(t_{1 / 2}\right)$ of $5.10 \mathrm{~h}$ (single substance) and $2.81 \mathrm{~h}$ (MR extract), respectively.

Differences for DMP between oral dosing of neat compound and MR extract are statistically significant $(p<$ 0.05 ) (Table 3). In comparison with the values after oral dosing of MR extract, peak concentration $\left(C_{\max }\right)$ for DMP decreased significantly $(p<0.05)$, while the time to peak $\left(T_{\max }\right)$ was clearly delayed $(p<0.01)$ and $t_{1 / 2}$ and $\mathrm{MRT}_{0-t}$ were prolonged $(p<0.05)$ following oral dosing of pure DMP. These differences indicated that the rate of absorption and elimination of DMP may be accelerated by MR extract, so that the $T_{\max }, t_{1 / 2}$, and $\mathrm{MRT}_{0-t}$ for DMP in MR extract were remarkably decreased. Three possible reasons may be responsible for the differences. First, the crude extract contained many other components besides DMP and some of them might induce drug interactions which promote the

Table 3 The pharmacokinetic parameters of DMP in rats after a single-dose administration ( $n=6)$

\begin{tabular}{lll}
\hline Parameters & $\begin{array}{l}\text { Oral neat compound } \\
\text { (mean } \pm \text { SD) }\end{array}$ & $\begin{array}{l}\text { Oral MR extract } \\
\text { (mean } \pm \text { SD) }\end{array}$ \\
\hline$T_{\max }(\mathrm{h})$ & $2.80 \pm 0.84$ & $0.85 \pm 0.14^{* *}$ \\
$C_{\max }(\mu \mathrm{g} / \mathrm{L})$ & $2232.30 \pm 426.73$ & $3407.51 \pm 1126.62^{*}$ \\
$t_{1 / 2 z}(\mathrm{~h})$ & $5.10 \pm 2.74$ & $2.81 \pm 0.80^{*}$ \\
$\mathrm{AUC}_{0-t}(\mu \mathrm{g} / \mathrm{L} \mathrm{h})$ & $21796.13 \pm 6691.11$ & $16311.98 \pm 4760.90$ \\
$\mathrm{AUC}_{0-\infty}(\mu \mathrm{g} / \mathrm{L} \mathrm{h})$ & $22064.56 \pm 6403.32$ & $16380.38 \pm 4770.11$ \\
$\mathrm{CLz}_{\mathrm{F}}(\mathrm{L} / \mathrm{h} / \mathrm{kg})$ & $0.05 \pm 0.01$ & $0.07 \pm 0.02$ \\
$\mathrm{MRT}_{0-t}(\mathrm{~h})$ & $8.03 \pm 2.20$ & $4.47 \pm 1.20^{*}$ \\
$\mathrm{MRT}_{0-\infty}(\mathrm{h})$ & $8.32 \pm 1.95$ & $4.56 \pm 1.23^{* *}$ \\
\hline$p_{0.05}^{* *}<0.01$ &
\end{tabular}

${ }^{*} p<0.05,{ }^{* *} p<0.01$ compared with neat compound 

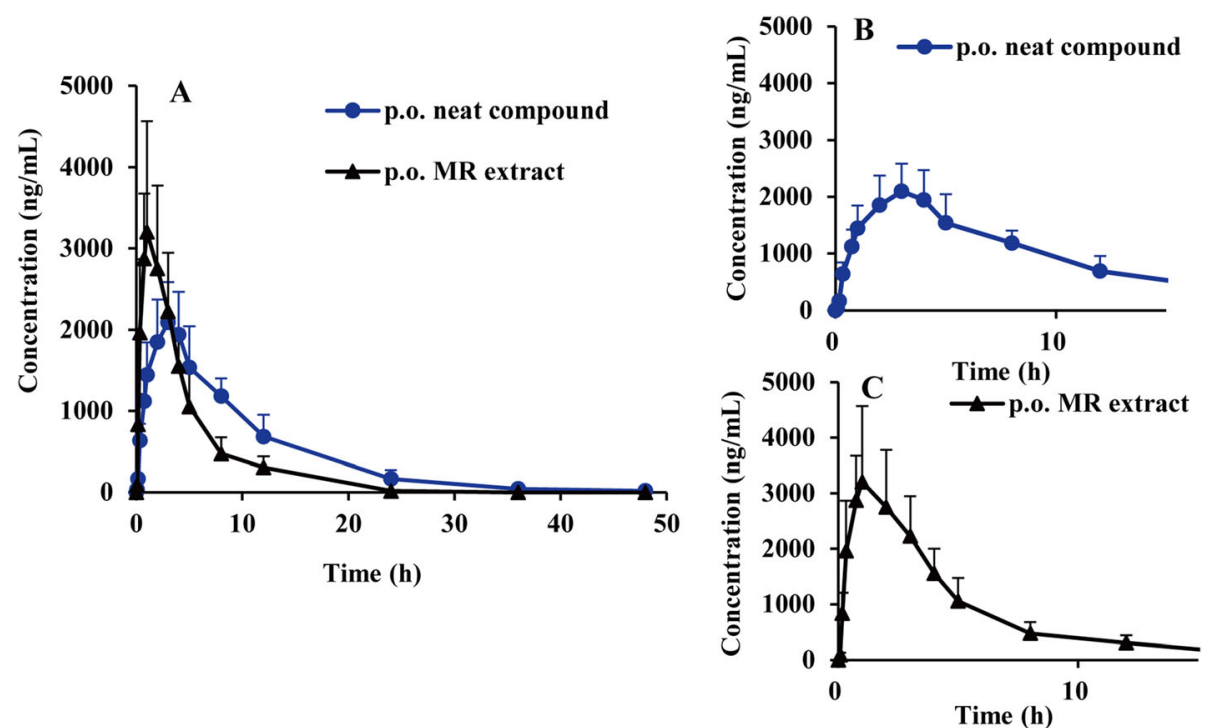

Fig. 3 Plasma concentration-time curves of DMP in rats following oral (p.o.) administration of pure DMP (1 mg/kg) and Menispermi Rhizoma extract (1.605 g/kg, dose equivalent to $1 \mathrm{mg} / \mathrm{kg}$ DMP): a comparison of two concentration-time curves, $\mathbf{b}$ pure DMP, and $\mathbf{c}$ Menispermi Rhizoma extract. Each value displays mean $\pm \mathrm{SD}, n=6$

absorption and elimination of DMP. Second, some coexistent components in MR extract may affect gastric emptying and gastrointestinal transit in rats and further affect the pharmacokinetic behavior of DMP. In addition, they may facilitate DMP absorption from the stomach and intestine such as changing intestine $\mathrm{pH}$ to inhibit DMP ionization, because DMP shows weak acidity (pKa 5.819) due to the occurrence of a phenolic hydroxyl functional group in the structure which could be ionized at physiological $\mathrm{pH}$. Third, some of the components might affect drug-metabolizing enzyme activity and intestinal micro-organisms which improve the metabolism and elimination rate of DMP. All the possible reasons above may have an impact on several aspects of absorption, distribution, metabolism, and excretion (ADME) of DMP. However, the reason for different pharmacokinetic profiles of DMP and the related action mechanism needs to be continuously and thoroughly investigated. From the results obtained, it could be concluded that oral administration of single substance had priority to that of MR extract due to its sustained pharmacological effects in vivo. The good absorption and slow elimination of pure DMP makes it possible to become a potentially useful anti-tumor agent. Thus, the present study lays an experimental foundation for further pharmacodynamics and druggability studies of DMP.

\section{Conclusions}

In summary, our investigation demonstrates that the newly developed HPLC-FLD method provides a sensitive assay for fast quantification of DMP in medicines and rat plasma. The present work is the first spectrofluorimetric approach for the assay of DMP. The assay provides greater efficiency and simplicity for analysis of biosample in terms of its fast and simple sample processing method and relatively short analysis time, which was successfully applied to the comparative pharmacokinetic study of DMP in rats following administration of neat compound and MR extract. These show that the new method would be a better option for conventional analysis and offers a practical, efficient, and sensitive approach for quantification of oxoisoaporphine alkaloids in complex biological matrices. This research provides an experimental basis for the evaluation of the preclinical safety of DMP.

\section{Abbreviations}

DMP: 6-O-Demethylmenisporphine; MR: Menispermi Rhizoma;

TCM: Traditional Chinese medicine; FLD: Fluorescence detection; IS: Internal standard

\section{Acknowledgements \\ Not applicable in this section.}

\section{Authors' contributions}

Jinxia Wei and Jia Shao designed the experiments and drafted the manuscript. Jinxia Wei and Yanan Li performed the experiments and data analysis. Yubo Li revised the manuscript. All authors read and approved the final manuscript.

\section{Funding}

This research was supported by the Natural Science Foundation of China (81703690, 81803356) and Natural Science Foundation of Tianjin (No 19JCQNJC12200).

Availability of data and materials

Data sharing is not applicable to this article.

Competing interests

The authors declare that there are no conflicts of interest. 


\section{Author details}

${ }^{1}$ School of Chinese Materia Medica, Tianjin University of Traditional Chinese Medicine, 312 Anshan West Road, Tianjin 300193, China. ${ }^{2}$ Department of Pharmacy, Tianjin First Center Hospital, 24 Fukang Road, Tianjin 300192, China.

Received: 19 June 2020 Accepted: 9 December 2020

Published online: 04 January 2021

\section{References}

Balla A, Cho KH, Kim YC, Maeng HJ. Simultaneous determination of Procainamide and $\mathrm{N}$-acetylprocainamide in rat plasma by ultra-high-pressure liquid chromatography coupled with a diode array detector and its application to a pharmacokinetic study in rats. Pharmaceutics. 2018;10:41.

Cheng JJ, Tsai TH, Lin LC. New alkaloids and cytotoxic principles from Sinomenium acutum. Planta Med. 2012;78:1873-7.

China Pharmacopoeia Committee. Pharmacopoeia of the People's Republic of China. Beijing: China Medical Science Press. 2015; Part 1: 99.

Huo LN, Jiang Z, Lei M, Wang XT, Guo XJ. Simultaneous quantification of Kirenol and ent-16ß,17-dihydroxy-kauran-19-oic acid from Herba Siegesbeckiae in rat plasma by liquid chromatography-tandem mass spectrometry and its application to pharmacokinetic studies. J Chromatogr B Analyt Technol Biomed Life Sci. 2013;937:18-24.

Li SS, Song X, Chai X, Wang YF. Chemical constituents from the rhizome of Menispermum dauricum DC. Nat Prod Res Dev. 2013;25:60-3.

Lou Y, Wu HY, Zheng JQ, He XY, Wu Z, Lu XY, Qiu YQ. Determination and pharmacokinetic study of skimmin by UHPLC-MS/MS in rat plasma. J Pharmaceut Biomed Anal. 2019; 179: doi.org/https://doi.org/10.1016/j.jpba.2019.112969.

Peng YR. Chemical constituents and pharmacological activities of whole herb of Menispermum dauricum DC. Pop Sci Technol. 2018;20:94-6.

Shao J, Shi CF, Wei JX, Li YX, Guo XJ. Chemical constituents from rhizome of Menispermum dauricum and their anti-hypoxic activities. China J Chin Mater Med. 2019:44:723-9.

Shen ZW, He KF, Xu MC, Zeng K, Pan J, Ou FT, Yao JB, Wang RW, Zeng S. Development and validation of a sensitive LC-MS/MS method for the determination of 6-hydroxykynurenic acid in rat plasma and its application to pharmacokinetics study. J Chromatogr B Analyt Technol Biomed Life Sci. 2019;1116:44-50.

Su Q, He J, Wang ZY, Lv L, Suo Y, Wang JJ, Zheng ZW, Huo CC, Li J. Intestinal anti-inflammatory effect of the rhizome extracts of menispermum dauricum DC. on trinitrobenzene sulfonic acid induced ulcerative colitis in mice. J Ethnopharmacol. 2016;193:12-20.

U.S. Food and Drug Administration, Guidance for industry: bioanalytical method validation. 2018. https://www.fda.gov/files/drugs/published/BioanalyticalMethod-Validation-Guidance-for-Industry.pdf.

Wang F, Qu L, Lu Q, Guo LJ. Effect of phenolic alkaloids from Menispermum dauricum on myocardial-cerebral ischemia/reperfusion injury in rabbits. Acta Pharmacol Sin. 2001;22:1130-4.

Wang JY, Sun S, Liu L, Yang WS. Induction of apoptosis in human cervical carcinoma HeLa cells with active components of Menispermum dauricum. Genet Mol Res. 2014;13:3545-52.

Wang P, Sun JB, Xu JY, Yan Q, Gao EZ, Qu W, Zhao YL, Yu ZG. Pharmacokinetics, tissue distribution and excretion study of dictamnine, a major bioactive component from the root bark of Dictamnus dasycarpus Turcz. (Rutaceae). J Chromatogr B Analyt Technol Biomed Life Sci. 2013;942-943:1-8.

Wei JX, Chen J, Liang XL, Guo XJ. Microwave-assisted extraction in combination with HPLC-UV for quantitative analysis of six bioactive oxoisoaporphine alkaloids in Menispermum dauricum DC. Biomed Chromatogr. 2016;30:241-8.

Wu D, Du J, Zhang Y, Su Y, Zhang H. Anti-tumor effects of phenolic alkaloids of menispermum dauricum on gastric cancer in vivo and in vitro. J. Cancer Res Ther. 2018;14:S505-11.

Yoon JH, Nguyen TTL, Duong VA, Chun KH, Maeng HJ. Determination of KD025 (SLX-2119), a selective ROCK2 inhibitor, in rat plasma by high-performance liquid chromatography-tandem mass spectrometry and its pharmacokinetic application. Molecules. 2020;25:1369.

Yu BW, Meng LH, Chen JY, Zhou TX, Cheng KF, Ding J, Qin GW. Cytotoxic oxoisoaporphine alkaloids from Menispermum dauricum. J Nat Prod. 2001;64: 968-70.

Yu YY, Shao J, Wei JX, Li YX, Li X, Li LZ, Gu J. Research progress of alkaloids and pharmacological activities in Menispermi Rhizoma. Chin Med Mat. 2019:42: 2455-63.
Zhang JY, Chen L, Sun JB. Oxoisoaporphine alkaloids, prospective antiAlzheimer's, anticancer and antidepression agents. ChemMedChem. 2018;13: 1262-74.

Zhao B, Chen Y, Sun X, Zhou M, Ding J, Zhan JJ, Guo LJ. Phenolic alkaloids from Menispermum dauricum rhizome protect against brain ischemia injury via regulation of GLT-1, EAAC1 and ROS generation. Molecules. 2012;17:2725-37.

Zhao JJ, Han X, Zhao X, Wang C, Li Q, Chen XH, Bi KS. A sensitive liquid chromatographic-mass spectrometric method for simultaneous determination of dehydroevodiamine and limonin from Evodia rutaecarpa in rat plasma. Anal Bioanal Chem. 2011;401:289-96.

\section{Publisher's Note}

Springer Nature remains neutral with regard to jurisdictional claims in published maps and institutional affiliations.

\section{Submit your manuscript to a SpringerOpen ${ }^{\circ}$ journal and benefit from:}

- Convenient online submission

- Rigorous peer review

- Open access: articles freely available online

- High visibility within the field

- Retaining the copyright to your article

Submit your next manuscript at $\boldsymbol{\nabla}$ springeropen.com 